\title{
Three-dimensional structure of the hepatitis B core antigen particle truncated at residue 154
}

\author{
LIU ShuYu $^{1 \dagger}$, HE Jian ${ }^{2 \dagger}$, LI KunPeng ${ }^{1}$, DAI AGuang ${ }^{1}$, CAI ChangJie ${ }^{3}$ \& ZHANG JingQiang ${ }^{1 *}$ \\ ${ }^{1}$ State Key Laboratory of Biocontrol, Life Sciences School, Sun Yat-sen University, Guangzhou 510275, China; \\ ${ }^{2}$ Guangzhou East Campus Lab Center, Sun Yat-sen University, Guangzhou 510006, China; \\ ${ }^{3}$ Third Affiliated Hospital of Sun Yat-sen University, Guangzhou 510080, China
}

Received December 14, 2009; accepted January 22, 2010; published online November 19, 2010

\begin{abstract}
The three-dimensional structure of recombinant hepatitis $\mathrm{B}$ core antigen $(\mathrm{HBcAg})$ particles truncated at residue 154 (HBcAg-154) was determined to $7.8 \AA$ resolution by cryo-electron microscopy (cryoEM) and computer reconstruction. The capsid of HBcAg-154 is mainly constituted by $\alpha$-helical folds, highly similar to that of HBcAg-149. The C-terminal region between residues 155 and 183 of the core protein is more crucial to the encapsidation of RNA, and the short C-terminal tail of $\mathrm{HBcAg}-154$ results in a nearly empty capsid.
\end{abstract}

HBcAg-154 particle, capsid, RNA, cryoEM

Citation: Liu S Y, He J, Li K P, et al. Three-dimensional structure of the hepatitis B core antigen particle truncated at residue 154. Sci China Life Sci, 2011, 54: 171-174, doi: 10.1007/s11427-010-4098-x

Hepatitis B virus (HBV) is a noncytopathic, enveloped, double-stranded DNA virus that causes acute and chronic hepatitis and hepatocellular carcinoma [1]. Among the estimated 350 million patients who are chronically infected with HBV, one million will die every year. HBV belongs to the genus Orthohepadnavirus in the family Hepadnaviridae. The family members replicate by reverse transcription of an RNA intermediate, the pre-genomic RNA (pgRNA) in the hepatocytes of their hosts. The HBV virion consists of an inner nucleocapsid, surrounded by a lipid envelope containing large, medium and small, three forms of surface proteins [2]. During virus assembly, the core protein polymerizes around a complex consisting of pgRNA and $\mathrm{P}$ protein to form an immature nucleocapsid. $\mathrm{P}$ protein is a multifunctional enzyme composed by a C-terminal RNase $\mathrm{H}(\mathrm{RH})$ domain, a reverse transcription (RT) domain, an $\mathrm{N}$-terminal protein (TP) domain and a middle spacer region [2]. The RNA is reverse transcribed into single-stranded DNA and concomi-

$\dagger$ Contributed equally to this work

*Corresponding author (email: 1sszhjq@mail.sysu.edu.cn) tantly digested by an RH domain, after which the partial second DNA strand is synthesized, formally declaring the maturation of the HBV nucleocapsid [3].

Both in vivo and in vitro, its core protein forms icosahedral capsids in two formations, respectively corresponding to triangulation numbers of $T=3$ and $T=4$ [4]. The core protein with 183 amino acids is divided into three parts: an $\mathrm{N}$-terminal core domain used to construct the major capsid (residues 1-140), a C-terminal tail indispensable for RNA/DNA binding (residues 150-183), and a 10-residue linker between them (residues 141-149), whose length determines the number ratio of $T=3 / T=4$ capsids [4].

The scaffold of $T=4$ hepatitis B core antigen ( $\mathrm{HBcAg}$ ) particles truncated at residue 149 (HBcAg-149) is mainly constituted by $\alpha$-helical folds, whose surface spikes are formed by bundles of four $\alpha$-helices, two from each contributing monomer [5-7]. Its inner space is empty without observing RNA for the lack of a C-terminal tail. The capsid structure of the full-length $\mathrm{HBcAg}$ particle (HBcAg-183) is similar to that of $\mathrm{HBcAg}-149$, while $\mathrm{HBcAg}-183$ contains 
large amounts of RNA [8,9]. The difference of RNA content indicates the significance of the C-terminal tail of the core protein in packaging RNA.

Here we report a three-dimensional structure of an $\mathrm{HBcAg}$ particle truncated at 154 (HBcAg-154) with a resolution of $7.8 \AA$ by cryo-electron microscopy (cryoEM), which highly resembles that of HBcAg-149 not only on the capsid surface, but also in the interior of the capsid. This result indicates that the $\mathrm{C}$-terminal tail of $\mathrm{HBcAg}-154$ is too short (only five residues, much less than that of $\mathrm{HBcAg}-183$ ) to encapsidate as many RNA molecules as $\mathrm{HBcAg}-183$.

\section{Materials and methods}

\subsection{Sample preparation and cryoEM}

The HBcAg-154 particles were kindly provided by Professor Shih Chiaho (University of Texas Medical Branch), whose production and purification were similar to the previous procedures [10].

Collecting cryoEM data from the $\mathrm{HBcAg}-154$ particles was performed as previously described [11]. 2-3 $\mu \mathrm{L}$ aliquot of $1.5 \mathrm{mg} \mathrm{mL}^{-1} \mathrm{HBcAg}$ particles was applied to a Quantifoil R 2/1 grid (Quantifoil Micro Tools Gmbh, Jena, Germany), quickly blotted with filter paper and plunged into liquid nitrogen-cooled liquid ethane so that the samples were embedded in a thin layer of vitreous ice across the holes of a carbon supporting film. Images were recorded at a magnification of $98000 \times$ (i.e., $59000 \times 1.66$, corresponding to $1.53 \AA$ pixel size on the specimen scale) at $300 \mathrm{kV}$ in an FEI G2 Polara cryo-electron microscope equipped with a TVIPS 16-megapixel CCD using an electron dose of 20 e $\AA^{-2} .150$ focal pair images were recorded. The first micrograph was a close-to-focus micrograph with an underfocus value of approximately $1.5 \mu \mathrm{m}$, and the second one was a far-from-focus image with an underfocus value approximately $2.5 \mu \mathrm{m}$.

\subsection{Three-dimensional reconstruction and visualization}

For three-dimensional reconstruction, selected micrographs in the appropriate defocus range, and without apparent specimen drift and charging, were used to select appropriate particles with the BOXER program of EMAN 1.8 [12]. Image processing and three-dimensional reconstruction were performed according to the established procedure [13] using the software package IMIRS [14] on an HP Workstation running Microsoft Windows XP.

Orientation estimation and refinement were done using both Fourier common lines $[15,16]$ and projection-matching methods [17]. The three-dimensional reconstruction was performed by the spherical harmonics method [18]. The defocus values of images were estimated from the positions of the contrast transfer function rings visible in the incoherently averaged Fourier transformations of particle im- ages [19], using the CTFIT program of the EMAN package [12]. Contrast transfer function correction and B-factor compensation were performed as previously described [20]. The focal-pair approach of orientation estimation was used, i.e., the orientation parameters were first determined from the far-from-focus pictures of the focal pairs and subsequently refined to a higher resolution using the close-to-focus pictures. Only the close-to-focus particle images were included in the final reconstruction [13]. Some particle images were eliminated based on phase residual and cross-correlation evaluations during orientation refinement and only about $70 \%-$ $80 \%$ of the initially selected particle images were included in the final $\mathrm{HBcAg}-154$ particle reconstructions.

The structures were segmented, displayed and fitted with core-149 crystal structures (PDB code: 1QGT) using UCSF Chimera [21]. For structural comparison and analysis, the volume structure of the $\mathrm{HBcAg}-149$ particle at $8 \AA$ resolution was made from its atomic structure using the "PDB2MRC" function of EMAN [12].

\section{Results and discussion}

\subsection{The cryoEM of HBcAg-154}

Under a cryo-electron microscope, the $\mathrm{HBcAg}-154$ particles have a typical hexagonal appearance whose outer surfaces are full of spikes (Figure 1). The overall diameters of most particles are $36 \mathrm{~nm}$ (99\% of all particles) across the tips of the spikes with a few of them having a diameter of about 32 $\mathrm{nm}$ (data not shown). The latter kind of particles has a $T=3$ icosahedral shell containing 180 subunits according to a previous report [22]. In our study, only the $T=4$ particles with a diameter of $36 \mathrm{~nm}$ were selected and used for the three-dimensional reconstruction. The final cryoEM map of the $\mathrm{HBcAg}-154$ particle at a resolution of $7.8 \AA$ was computed from images of 1124 individual particles taken from 150 micrographs.

\subsection{The surface features of the capsid}

From the reconstruction map of $\mathrm{HBcAg}-154$, we observed

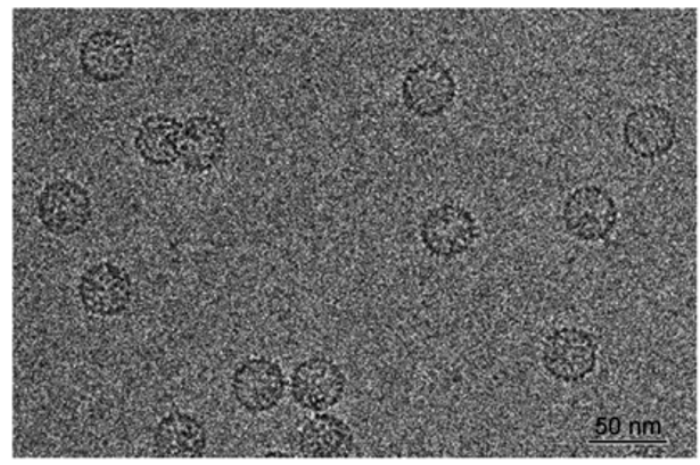

Figure 1 Cryo-electron micrograph of HBcAg-154 particles. 
120 spikes on the shell surface from the outer view, as well as numerous holes which were distributed on a two-fold axis (diameter 12-15 $\AA$ ), three-fold axis (diameter $14 \AA$ ), quasi-three-fold axis (diameter $14 \AA$ ) and five-fold axis (diameter $3 \AA$ ). The length of each spike was around $22 \AA$, as a classical symbol of HBV (Figure 2).

Overall, the particle shows that a $T=4$ icosahedral shell is very similar to that computed from $\mathrm{HBcAg}-149$ [5], both containing 240 subunits clustered in 120 dimers. The atomic model of HBcAg-149 particle also fits very well into this map with an average-map-value of 85.25 in Chimera (Figure $3 \mathrm{~A}$ ). The boundaries of each dimer unit are clearly visible in all regions, as well as the position of subunits in every dimer: Two subunits associate to give a compact dimer in which the two $\alpha$-helices pack to form a four-helix bundle (Figure $3 \mathrm{~B}$ and $\mathrm{C}$ ). The well-fitting result indicates our cryoEM structure is accurate.
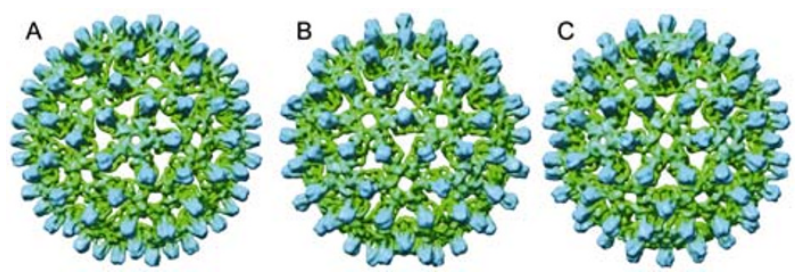

Figure 2 Three-dimensional map of $\mathrm{HBcAg}-154$ particles, represented as a shaded surface. A-C, The surface views along the icosahedral five- (A), three- (B), and two-fold (C) axes.
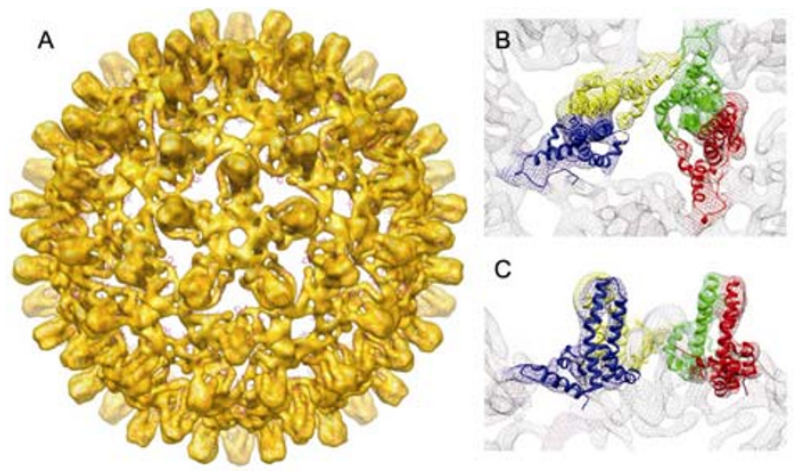

Figure 3 Detailed view of the cryoEM map of $\mathrm{HBcAg}-154$. A, The atomic structure of $\mathrm{HBcAg}-149$ is embedded as a purple ribbon diagram. B and $\mathrm{C}$, The atomic structure of core-149 is fitted into the cryoEM map represented by mesh lines. The atomic model of core-149 is shown using ribbons with colors corresponding to different subunits.
The close consistency between these two structures indicates that no gross alteration has occurred in the outer protein shell as a result of the increase of basic C-terminal tails. It also indicates that the $\mathrm{N}$-terminal core domain of the core protein (residues 1-140) is used to construct the major capsid, which exists both in HBcAg-154 and 149. There is no structural alteration of the capsid without changing the core domain.

\subsection{The inner structure of $\mathrm{HBcAg-154}$}

The inner structure of $\mathrm{HBcAg}-154$ was highly similar to that of HBcAg-149 [5-7] (Figure 4). The RNA density was not observed inside the capsid while a strong shell of RNA was present between radii 90-125 $\AA$ in HBcAg-183 [9]. As one kind of $\mathrm{HBcAg}$ particle constructed by the entire core protein, $\mathrm{HBcAg}-183$ encapsidates large amounts of RNA in the capsid. In addition, $\mathrm{HBcAg}$ particles truncated between residues 154 and 183, such as HBcAg-164, 169, 171 and 173, also contain obvious RNA densities inside for their basic C-terminal tails which are longer than that of HBcAg-154 [10].

Arginine is the major amino acid responsible for the binding of RNA in the basic C-terminal tail. However, $\mathrm{HBcAg}-154$ has only three arginines (R150, R152 and R154) in its short tail, less than those truncated $\mathrm{HBcAg}$ particles mentioned above (i.e., HBcAg-164 and 173) (Figure 5). Thus we believe both HBcAg-149 and 154 lack enough arginines to bind large amounts of RNA, resulting in the nearly empty capsids. The residue $154-183$ region in the C-terminal tail is an important factor in the encapsidation of RNA, which is rich in arginines.
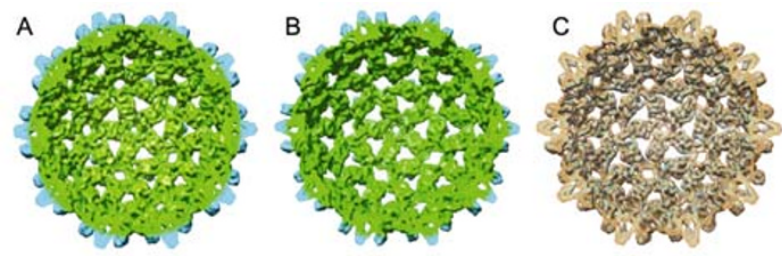

Figure 4 The inner structure of capsids viewed along a five-fold axis. A, The cryoEM structure of $\mathrm{HBcAg}-154$. B, The volume structure of HBcAg-149 at $8 \AA$ resolution has been filtered from the atomic structure of $\mathrm{HBcAg}$-149. C, Superposition of the HBcAg-154 (orange) and 149 (blue) maps.

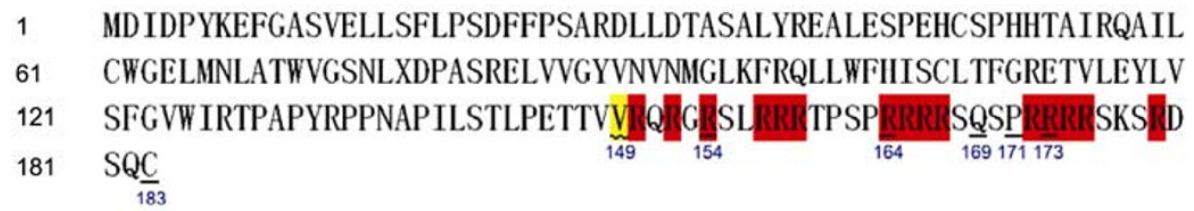

Figure 5 The protein sequence of the core protein. The V149 is marked as a yellow background while the arginines in the C-terminal tail have a red background. The residues of 149, 154, 164, 169, 171, 173 and 183 are also underlined 
In summary, we gained the high-resolution structure of the HBcAg-154 particle through cryoEM reconstruction. The three-dimensional map reveals a protein architecture highly similar to that of HBcAg-149 [5-7]. The inner space of the HBcAg-154 particle is also nearly empty, indicating the minimum volume of RNA inside [10]. This is an outcome because its short C-terminal tail lacks enough arginines to package RNA.

This work was supported by special funds of the National Natural Science Foundation of China (Grant No. 10274106). We thank Professor Shih Chiaho (University of Texas Medical Branch) for kindly providing the HBcAg particle samples used for cryoEM, as well as Professor Zhou Z. Hong (The University of Texas Medical School at Houston \& the University of California at Los Angeles) for warmheartedly providing cryoEM equipments related to sample preparation and imaging.

1 Chisari F V, Ferrari C. Hepatitis-B virus immunopathogenesis. Annu Rev Immunol, 1995, 13: 29-60

2 Beck J, Nassal M. Hepatitis B virus replication. World J Gastroenterol, 2007, 13: 48-64

3 Ganem D. Hepadnaviridae and their replication. In: Fields B N, Knipe D M, Howley P M, eds. Fields Virology. Philadelphia: Raven Press, 1996. 2703-2737

4 Wingfield P T, Stahl S J, Williams R W, et al. Hepatitis core antigen produced in Escherichia coli: Subunit composition, conformational analysis, and in vitro capsid assembly. Biochemistry, 1995, 34: 4949-4932

5 Böttcher B, Wynne S A, Crowther R A. Determination of the fold of the core protein of hepatitis B virus by electron cryomicroscopy. Nature, 1997, 386: 88-91

6 Conway J F, Cheng N, Zlotnick A, et al. Visualization of a 4-helix bundle in the hepatitis B virus capsid by cryo-electron microscopy. Nature, 1997, 386: 91-94

7 Wynne S A, Crowther R A, Leslie A G. The crystal structure of the human hepatitis B virus capsid. Mol Cell, 1999, 3: 771-780

8 Dryden K A, Wieland S F, Whitten-Bauer C, et al. Native hepatitis B virions and capsids visualized by electron cryomicroscopy. Mol Cell,
2006, 22: 843-850

9 Roseman A M, Berriman J A, Wynne S A, et al. A structural model for maturation of the hepatitis B virus core. Proc Natl Acad Sci USA, 2005, 102: 15821-15826

10 Le Pogam S, Chua P K, Newman M, et al. Exposure of RNA templates and encapsidation of spliced viral RNA are influenced by the arginine-rich domain of human hepatitis B virus core antigen (HBcAg 165-173). J Virol, 2005, 79: 1871-1887

11 Yu X, Jin L, Zhou Z H. 3.88 Å structure of cytoplasmic polyhedrosis virus by cryo-electron microscopy. Nature, 2008, 453: 415-419

12 Ludtke S J, Baldwin P R, Chiu W. EMAN: Semi-automated software for high resolution single particle reconstructions. J Struct Biol, 1999, 128: 82-97

13 Zhou Z H, Chiu W. Determination of icosahedral virus structures by electron cryomicroscopy at subnanometer resolution. Adv Protein Chem, 2003, 64: 93-124

14 Liang Y, Ke E Y, Zhou Z H. IMIRS: A high-resolution 3D reconstruction package integrated with a relational image database. J Struct Biol, 2002, 137: 292-304

15 Crowther R A. Procedures for three-dimensional reconstruction of spherical viruses by Fourier synthesis from electron micrographs. Philos Trans R Soc Lond B Biol Sci, 1971, 261: 221-230

16 Fuller S D. The $T=4$ envelope of Sindbis virus is organized by interactions with a complementary $T=3$ capsid. Cell, 1987, 48: 923-934

17 Baker T S, Cheng R H. A model-based approach for determining orientations of biological macromolecules imaged by cryoelectron microscopy. J Struct Biol, 1996, 116: 120-130

18 Liu H, Cheng L, Zeng S, et al. Symmetry-adapted spherical harmonics method for high-resolution 3D single-particle reconstructions. J Struct Biol, 2008, 161: 64-73

19 Zhou Z H, Hardt S, Wang B, et al. CTF determination of images of ice-embedded single particles using a graphics interface. J Struct Biol, 1996, 116: 216-222

20 Zhou Z H, Chen D H, Jakana J, et al. Visualization of tegumentcapsid interactions and DNA in intact herpes simplex virus type 1 virions. J Virol, 1999, 73: 3210-3218

21 Pettersen E F, Goddard T D, Huang C C, et al. UCSF Chimera-a visualization system for exploratory research and analysis. J Comput Chem, 2004, 25: 1605-1612

22 Crowther R A, Kiselev N A, Bottcher B, et al. Three-dimensional structure of hepatitis-B virus core particles determined by electron cryomicroscopy. Cell, 1994, 77: 943-950

Open Access This article is distributed under the terms of the Creative Commons Attribution License which permits any use, distribution, and reproduction in any medium, provided the original author(s) and source are credited. 